\title{
Synthesis and Properties of Two Dimensional Doped Transition Metal Dichalcogenides
}

\author{
Aram Yoon, Zonghoon Lee* \\ School of Materials Science and Engineering, Ulsan National Institute of Science and Technology (UNIST), UIsan 44919, Korea
}

*Correspondence to:

Lee $\mathrm{Z}$,

Tel: $+82-52-217-2327$

Fax: +82-52-217-2309

E-mail: zhlee@unist.ac.kr

Received March 27, 2017

Revised March 27, 2017

Accepted March 27, 2017
Since graphene was discovered in 2004, two-dimensional (2D) materials have been actively studied. Especially, 2D transition metal dichalcogenides (TMDs), such as $\mathrm{MoS}_{2}$ and $\mathrm{WS}_{2}$, have been the subject of significant research because of their exceptional optical, electrical, magnetic, catalytic, and morphological properties. Therefore, these materials are expected to be used in a variety of applications. Furthermore, tuning the properties of TMDs is essential to improve their performance and expand their applications. This review classifies the various doping methods of 2D TMDs, and it summarizes how the dopants interact with the materials and how the performance of the materials improves depending on the synthesis methods and the species of the dopants.

Key Words: Two-dimensional materials, Transition metal dichalcogenides, Doping, Chemical vapor transport, Chemical doping

\section{INTRODUCTION}

Two-dimensional (2D) materials have been intensively studied since graphene was discovered by Novoselov et al. (2004). The isolation of graphene from graphite demonstrated the possibility of isolating other stable monolayer or few-layer 2D materials, such as hexagonal boron nitride, silicene, and transition metal dichalcogenides (TMDs). The representative atomic structures of some 2D materials are shown in Fig. 1 (Tedstone et al., 2016). TMDs are generally denoted as $\mathrm{MX}_{2}$, where $\mathrm{M}$ is the transition metal ( $\mathrm{Mo}, \mathrm{W}, \mathrm{Ti}, \mathrm{V}$, etc.) and $\mathrm{X}$ is a chalcogen ( $\mathrm{S}, \mathrm{Se}, \mathrm{Te}$, etc.); these atoms are layered as $\mathrm{X}-\mathrm{M}-\mathrm{X}$ units by van der Waals bonding (Tedstone et al., 2016). Theoretically, there are 88 combinations of TMDs, but it was discovered using density functional theory (DFT) that only 44 stable monolayer or few-layer TMDs can exist stably (Ataca et al., 2012). The stability of few-layer TMDs is important because these materials have layer-dependent properties. It is known that the bulk may have an indirect bandgap $\left(\mathrm{E}_{\mathrm{g}}=1.29\right.$ $\mathrm{eV})$ and the monolayer may have a direct bandgap $\left(\mathrm{E}_{\mathrm{g}}=1.90\right.$ eV) for $\mathrm{MoS}_{2}$ (Wang et al., 2012). The exceptional bandgap structures of monolayer or few-layer TMDs endow them with enhanced optical and electrical properties. Therefore, 2D TMDs are expected to be useful for various applications, such as field effect transistors (FETs), optoelectronic devices, topological insulators, and electrocatalysts (Wang et al., 2015). In particular, $2 \mathrm{D}$ TMDs have attractive prospects as tunable high-performance channel materials for FETs (Mak et al., 2013).

To extend the potential of 2D TMDs, tuning of their properties is essential; diverse methods to accomplish this have been proposed, such as inducing strain, intercalation, controlling the layer number, and doping. In this article, the doping methods and the resulting enhanced properties of 2D TMDs are reviewed. The synthesis methods and the dopant atoms greatly influence the morphologies and properties of these materials. Therefore, selecting both an appropriate synthesis method and an appropriate species of dopant are critical.

\section{SYNTHESIS AND PROPERTIES OF DOPED TMDS}

\section{Chemical Vapor Transport}

Chemical vapor transport (CVT) is one of the most widely

(a) This is an open-access article distributed under the terms of the Creative Commons Attribution Non-Commercial License (http://creativecommons.org/licenses/by-nc/4.0) which permits unrestricted noncommercial use, distribution, and reproduction in any medium, provided the original work is properly cited.

Copyrights () 2017 by Korean Society of Microscopy 
A

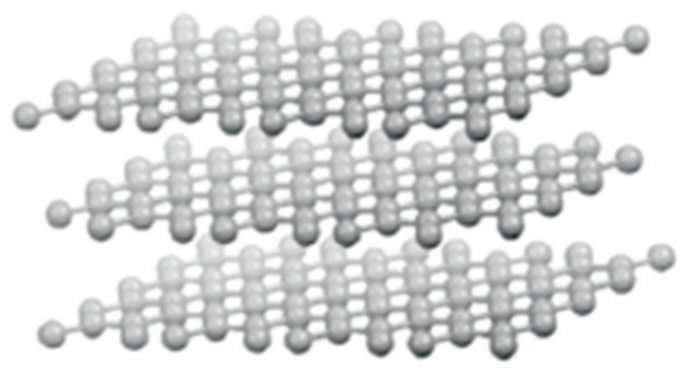

B

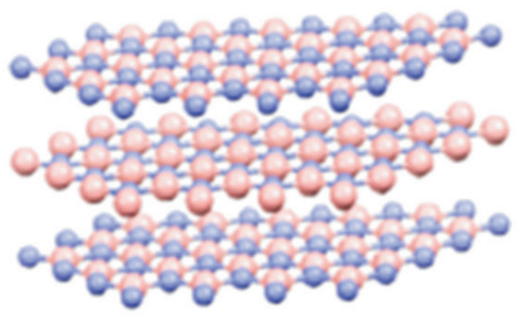

C

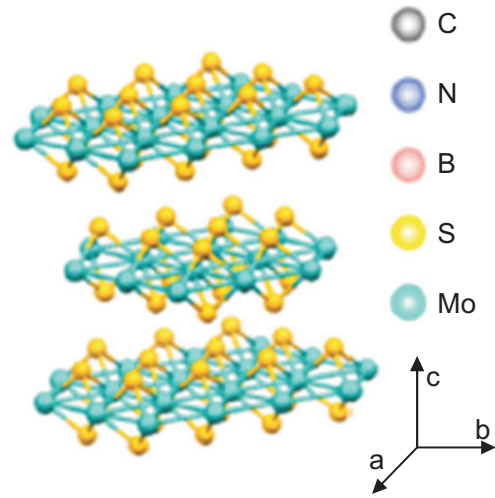

Fig. 1. Crystal structures of layered materials. (A) Graphite, (B) hexagonal boron nitride, (C) molybdenum disulfide. Reprinted from Tedstone et al. (2016) (Chem. Mater. 28, 1965-1974) with permission of American Chemical Society.
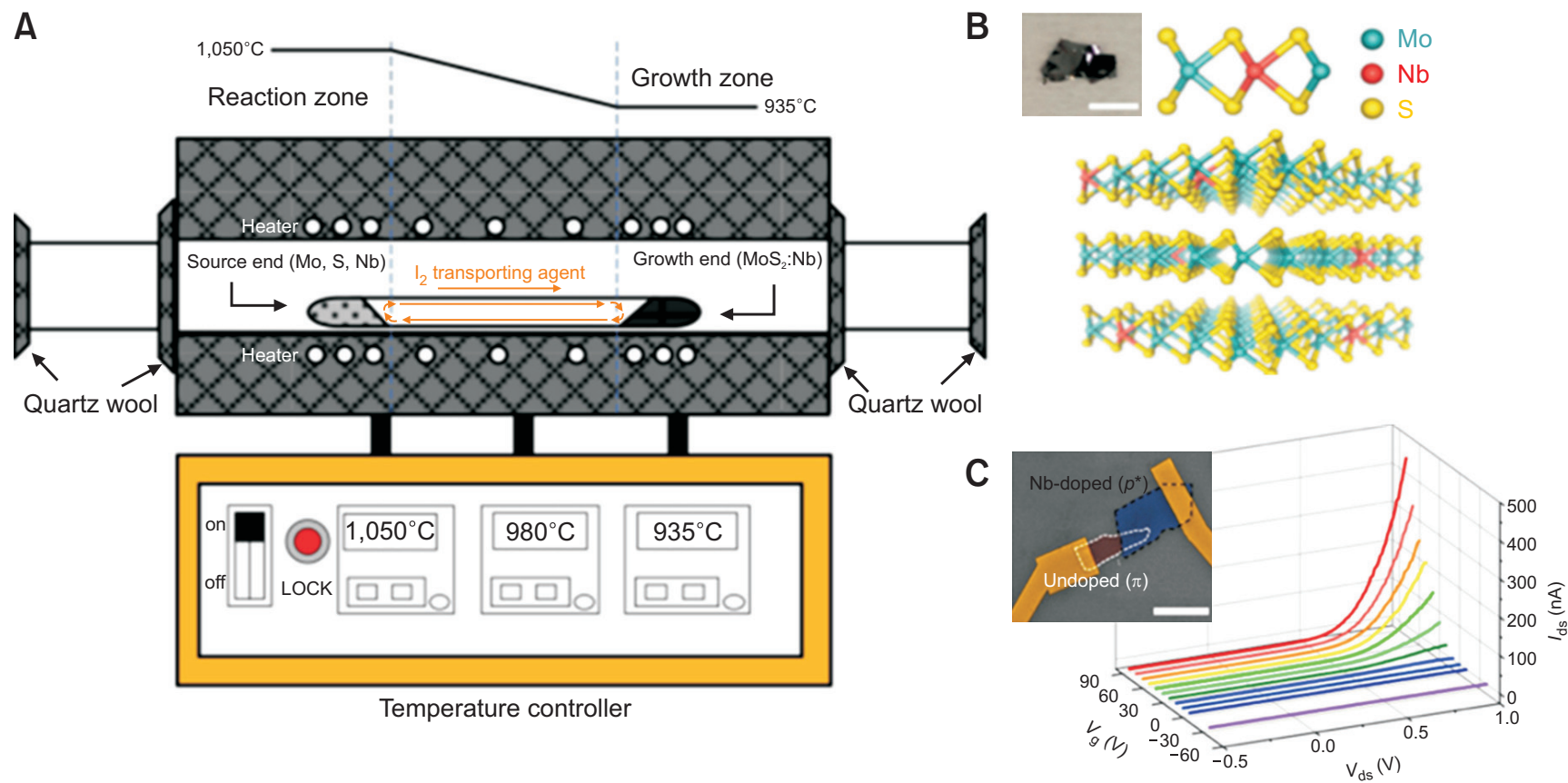

Fig. 2. Nb-doped $\mathrm{MoS}_{2}$ crystal. (A) Schematic of chemical vapor transport with a three-zone furnace setup. (B) Crystal structure in which $\mathrm{Nb}$ is doped in the substitutional sites of Mo. (C) I-V characteristics of van der Waals p-n junctions with Nb-doped $\operatorname{MoS}_{2}(60 \mathrm{~nm})$ and undoped MoS $2(4 \mathrm{~nm})$ which measured under variable gate voltages and drain-source voltages. Reprinted from Suh et al. (2014) (Nano Lett. 14, 6976-6982) with permission of American Chemical Society.

used methods for synthesizing 2D materials and doping TMDs. CVT was first popularized by Schäfer, and it involves reactions in several temperature zones. The precursors are volatilized and transported with the transport agents to the reaction zone. The reacted and decomposed precursors are deposited on the substrate in the growth zone in crystalline form (Binnewies et al., 2013). Optimizing the temperature of reaction zone and growth zone on the basis of the used materials is essential for successful synthesis. In addition, the transport agent, generally a halogen or halogen compounds that reacts with the precursors and forms gaseous compounds should be carefully selected.

Suh et al. (2014) researched niobium (Nb) doping on molybdenum disulfide $\left(\mathrm{MoS}_{2}\right)$ as a p-type. The experimental setup is shown in Fig. 2A. In the closed ampoule, the elemental precursor powders, $\mathrm{Mo}, \mathrm{S}$, and $\mathrm{Nb}$, are prepared at the reaction zone with a stoichiometric ratio of 1:2:0.005 when the target dopant concentration is $0.5 \%$. The temperatures of the reaction zone and the growth zone are $1,050^{\circ} \mathrm{C}$ and $935^{\circ} \mathrm{C}$, respectively. In addition, iodine gas $\left(\mathrm{I}_{2}\right)$ is used as the transport agent for this synthesis. Thus, $\mathrm{Nb}$ atoms are doped on $\mathrm{MoS}_{2}$ at the substitutional sites, as shown in Fig. 2B, and 
it leads to a degenerating hole density of around $3 \times 10^{19} \mathrm{~cm}^{-3}$. Additionally, van der Waals $p-n$ homojunctions are fabricated with the $\mathrm{Nb}$-doped and the undoped $\mathrm{MoS}_{2}$ flakes, which enables gate-tunable current rectification. The results are shown in Fig. 2C. The fabricated FET using Nb-doped and undoped $\mathrm{MoS}_{2}$ flakes exhibits enhanced current densities and tunable junction currents.

Lin et al. (2014) demonstrated the doping of rhenium (Re) and gold $(\mathrm{Au})$ on monolayer $\mathrm{MoS}_{2}$ via CVT growth. The atomic structures of the doped $\mathrm{MoS}_{2}$ sheets were observed by scanning transmission electron microscopy (STEM). Fig. $3 \mathrm{~A}$ and $\mathrm{F}$ show $\mathrm{Z}$-contrast annular dark-field (ADF) images of Re-doped $\mathrm{MoS}_{2}$ and Au-doped $\mathrm{MoS}_{2}$, respectively. The dopants appear in the image as brighter contrast spots because of its heavier atomic weight. In Fig. 3B-D and G-I, sequential $\mathrm{ADF}$ images show the migration of each dopants, respectively. The immobile Re dopants are doped at substitutional Mo sites, and the mobile Re dopants simply bond with sulfur (S) atoms. However, the Au dopants bond with $S$ atoms and exists as adatoms, which have greater mobility under the electron beam. The predicted atomic structures of Re- and Au-doped $\mathrm{MoS}_{2}$ are illustrated in Fig. 3E and J, respectively. These atomic models were also confirmed by DFT.

\section{In Situ Doping in Chemical Vapor Deposition}

Chemical vapor deposition (CVD) is one of the most commonly used methods to synthesize 2D materials (Park \& Sudarshan, 2001). The volatile precursors react and decompose under low pressure and high temperature, and the products are deposited on the substrate. To prevent the creation of byproducts, the gas flow in the CVD reaction chamber should be well controlled.

Zhang et al. (2015) fabricated manganese (Mn)-doped $\mathrm{MoS}_{2}$ by in situ doping using the CVD method. The pristine and $\mathrm{Mn}$-doped $\mathrm{MoS}_{2}$ monolayer were grown by vaporization of the applied sources; a schematic of the CVD furnace setup is illustrated in Fig. 4A. Two milligrams of molybdenum oxide $\left(\mathrm{MoO}_{3}\right)$ is heated to $725^{\circ} \mathrm{C}$ at the growth end of the furnace, and $700 \mathrm{mg}$ of sulfur powder and $0.1 \mathrm{mg}$ dimanganese decacarbonyl $\mathrm{Mn}_{2}(\mathrm{CO})_{10}$ powder is vaporized in the upstream region of the furnace, and these two upstream regions are heated to $300^{\circ} \mathrm{C}$ and $70^{\circ} \mathrm{C}$, respectively. The vaporized $\mathrm{Mn}$ and $\mathrm{S}$ are transferred to the growth end of the furnace with argon (Ar) gas. The doped $\mathrm{Mn}$ is readily distinguished by high-angle annular dark field scanning transmission electron microscopy (HAADF-STEM) at the domain boundary of $\mathrm{MoS}_{2}$ directly grown on a TEM grid via CVD, as shown in Fig. $4 \mathrm{~B}$ and $\mathrm{C}$. However, the success rate of the incorporation of $\mathrm{Mn}$ in $\mathrm{MoS}_{2}$ was significantly influenced by the species of the substrate, which included graphene and a traditional insulating substrate. Inert substrates such as graphene enabled incorporation of dopants in TMDs. However, traditional substrates, which have reactive surfaces, disrupted the incorporation of the dopants in $\mathrm{MoS}_{2}$ and induced defects. In addition, Fig. $4 \mathrm{D}$ and $\mathrm{E}$ demonstrate modifications of the bandgap structure through in situ doping via photolumines-
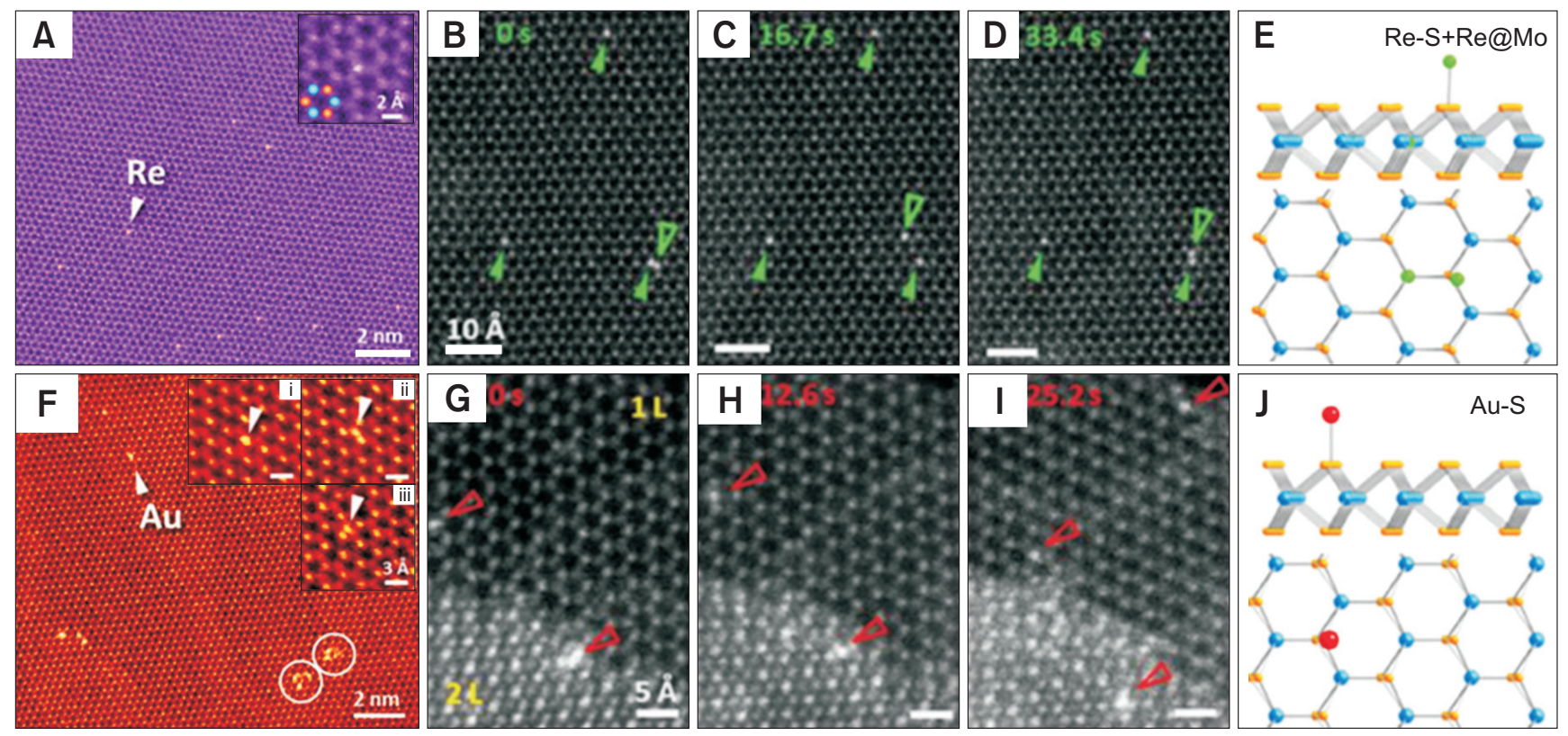

Fig. 3. (A) Annular dark-field (ADF) image of monolayer Re-doped $\mathrm{MoS}_{2}$ and (B-D) sequential ADF images of monolayer Re-doped MoS ${ }_{2}$ The hollow arrows indicate Re-S adatoms, and the closed arrows indicate doped Re at substitutional Mo sites. (F) ADF image of monolayer Au-doped MoS ${ }_{2}$ and (G-I) sequential ADF images of monolayer Au-doped $\mathrm{MoS}_{2}$ at the Au-S sites. (E) and (J) Atomic models of Re-doped and Au-doped MoS ${ }_{2}$, respectively. Reprinted from Lin et al. (2014) (Adv. Mater. 26, 2857-2861) with permission of Advanced Materials. 
A

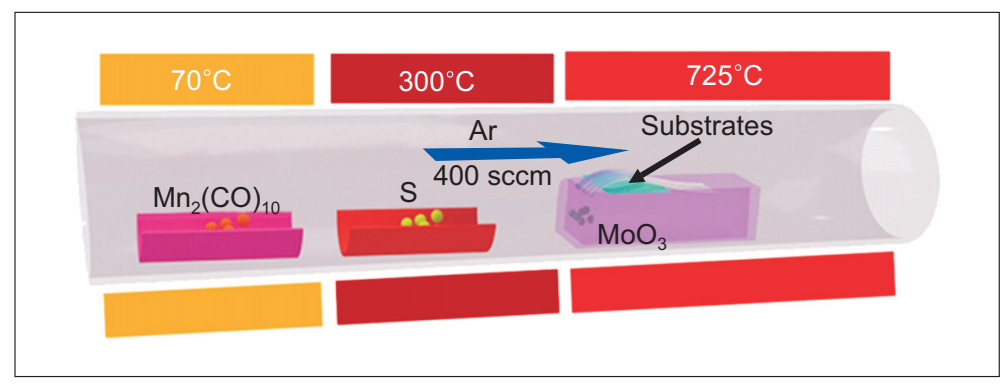

B

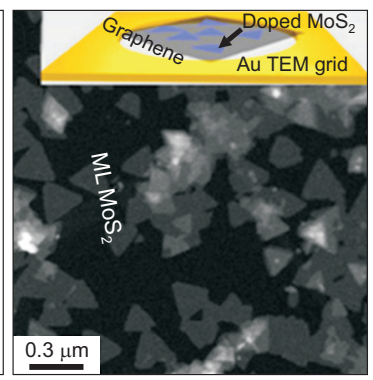

C

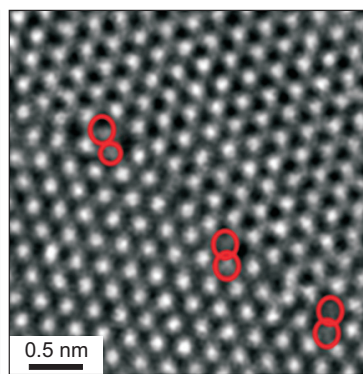

D

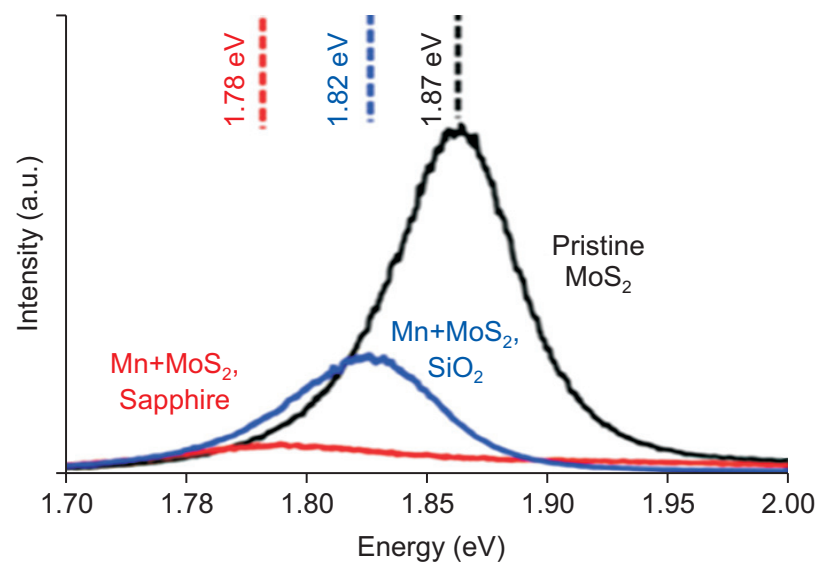

$E$

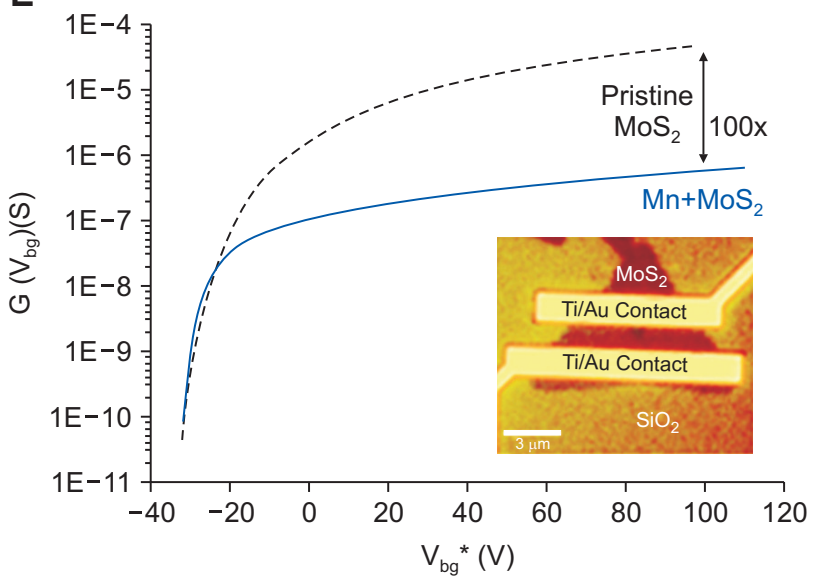

Fig. 4. (A) Schematic of chemical vapor deposition with a three-zone furnace for synthesizing pristine and Mn-doped MoS 2 crystals. (B) Transmission electron microscopy (TEM) image of Mn-doped $\mathrm{MoS}_{2}$ directly grown on a suspended graphene TEM grid and (C) high-angle annular dark field scanning TEM image of Mn-doped $\mathrm{MoS}_{2}$, where the Mn dopants are clearly distinguished at the $\mathrm{MoS}_{2}$ domain boundary. (D) Photoluminescence spectra of pristine and doped $\mathrm{MoS}_{2}$ on each substrate. (E) Conductance versus back-gate voltage measurement, indicating that Mn doping lowers the saturation conductance. Reprinted from Zhang et al. (2015) (Nano Lett. 15, 6586-6591) with permission of American Chemical Society.

cence (PL) spectra and saturation conductance investigations, respectively.

Gao et al. (2016) demonstrated the synthesis of Nb-doped tungsten disulfide $\left(\mathrm{WS}_{2}\right)$ via in situ CVD method. The precursor, $200 \mathrm{mg}$ of tungsten oxide $\left(\mathrm{WO}_{3}\right)$ was vaporized at approximately $900^{\circ} \mathrm{C}$ at the end of the CVD furnace. Then, $200 \mathrm{mg}$ of sulfur for sulfurization and $1 \mathrm{mg}$ of $\mathrm{NbCl}_{5}$ for $\mathrm{Nb}$ doping were placed upstream with a heating belt under $100^{\circ} \mathrm{C}$, as shown in Fig. $5 \mathrm{~A}$. The $\mathrm{NbCl}_{5}$ and the sulfur were provided simultaneously when the temperature of the CVD furnace reached $900^{\circ} \mathrm{C}$. Then, $\mathrm{Nb}$-doped $\mathrm{MoS}_{2}$ was grown on a sapphire substrate under $120 \mathrm{mTorr}$ for $30 \mathrm{~min}$ with ultrahigh purity Ar gas. The doping of transition metals such as $\mathrm{Nb}$ at the substitutional sites was relatively stable as Fig. 5D shown. In addition, the doped TMDs retained the semiconducting properties of the pristine monolayer as well as other attractive characteristics, such as direct-bandgap PL. It was verified by both experiments and DFT calculations.

\section{Aerosol-Assisted Chemical Vapor Deposition}

Aerosol-assisted chemical vapor deposition (AACVD) is a
CVD method that is usually utilized for non-volatile precursors (Hwang, 2016). The precursors are dissolved in solvent, and the aerosol form is generated by sonication; then, the created precursor mist is transported with a carrier gas to the reaction chamber. Finally, the adsorbed precursors react and deposit on the substrate relatively uniformly, and the byproducts are diffused.

Lewis et al. (2015) fabricated chromium (Cr)-doped $\mathrm{MoS}_{2}$ via AACVD. The precursor was prepared as a solution by mixing tetrakis(diethyldithiocarbamato)molybdenum(IV) $\left(\mathrm{MoL}_{4}\right)$ and tris(diethyldithiocarbamato)chromium(III) $\left(\mathrm{CrL}_{3}\right)$ and dissolving the mixture in $25 \mathrm{~mL}$ of tetrahydrofuran (THF). Ar gas was used to transport the precursor mist to a tube containing the glass substrate at $450^{\circ} \mathrm{C}$ or $500^{\circ} \mathrm{C}$ for $100 \mathrm{~min}$. A simple schematic of AACVD is illustrated in Fig. 6. During the synthesis, Cr-doped $\mathrm{MoS}_{2}$, pristine $\mathrm{MoS}_{2}$, and a small amount of $\mathrm{Cr}-\mathrm{S}$ were generated. Finally, the nanosheets of $\mathrm{Cr}-$ doped $\mathrm{MoS}_{2}$ were produced by liquid-phase exfoliation. The $\mathrm{Cr}$ dopants were deposited uniformly throughout the $\mathrm{MoS}_{2}$, as shown in Fig. 6, in which the surface of the Cr-doped $\mathrm{MoS}_{2}$ is observed via STEM energy dispersive X-ray spectroscopy 
A

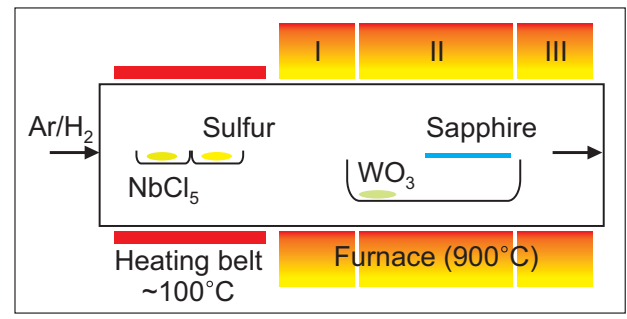

B

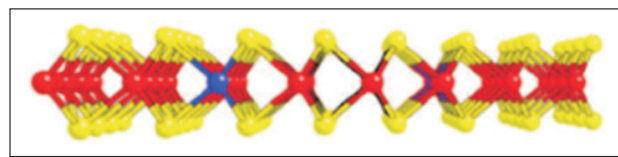

C

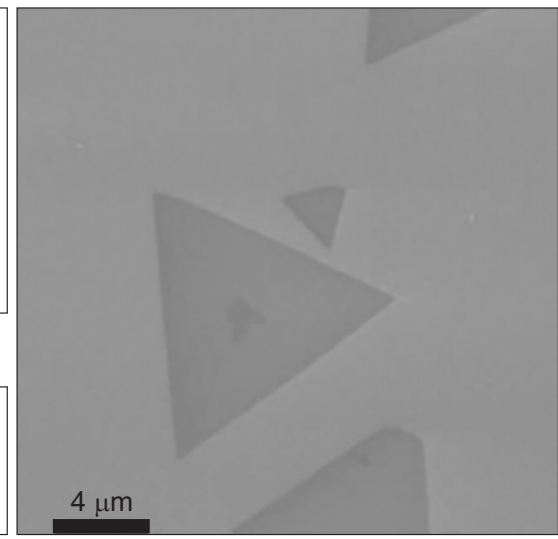

D

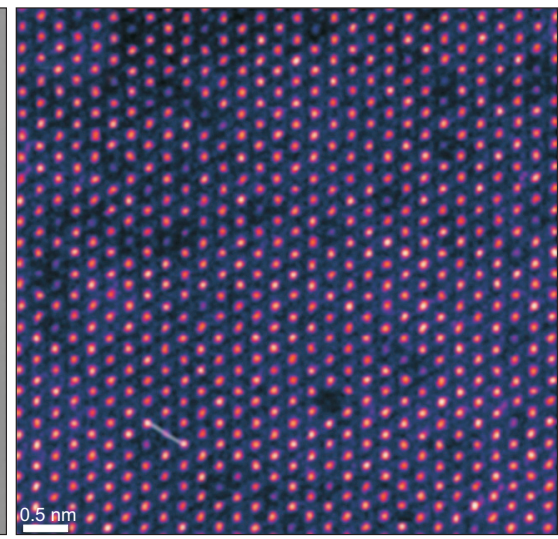

Fig. 5. Substitutional doping of $\mathrm{Nb}$ on monolayer $\mathrm{WS}_{2}$ via in situ chemical vapor deposition (CVD). (A) Schematic of the CVD furnace setup. (B) Crosssection schematic of monolayer $\mathrm{WS}_{2}$ with $\mathrm{Nb}$ doping (red, $\mathrm{W}$; blue, Nb; yellow, sulfur). (C) Scanning electron microscopy image of CVD-grown monolayer $\mathrm{Nb}$-doped $\mathrm{WS}_{2}$ triangles, (D) aberration-corrected Z-contrast scanning transmission electron microscopy image of monolayer $\mathrm{Nb}$-doped WS $\mathrm{C}_{2}$. The yellow and purple dots represent $\mathrm{W}$ and $\mathrm{Nb}$, respectively. The drawn white line represents the alignment of W-Nb-W. Reprinted from Gao et al. (2016) (Adv. Mater. 28, 9735-9743) with permission of Advanced Materials.

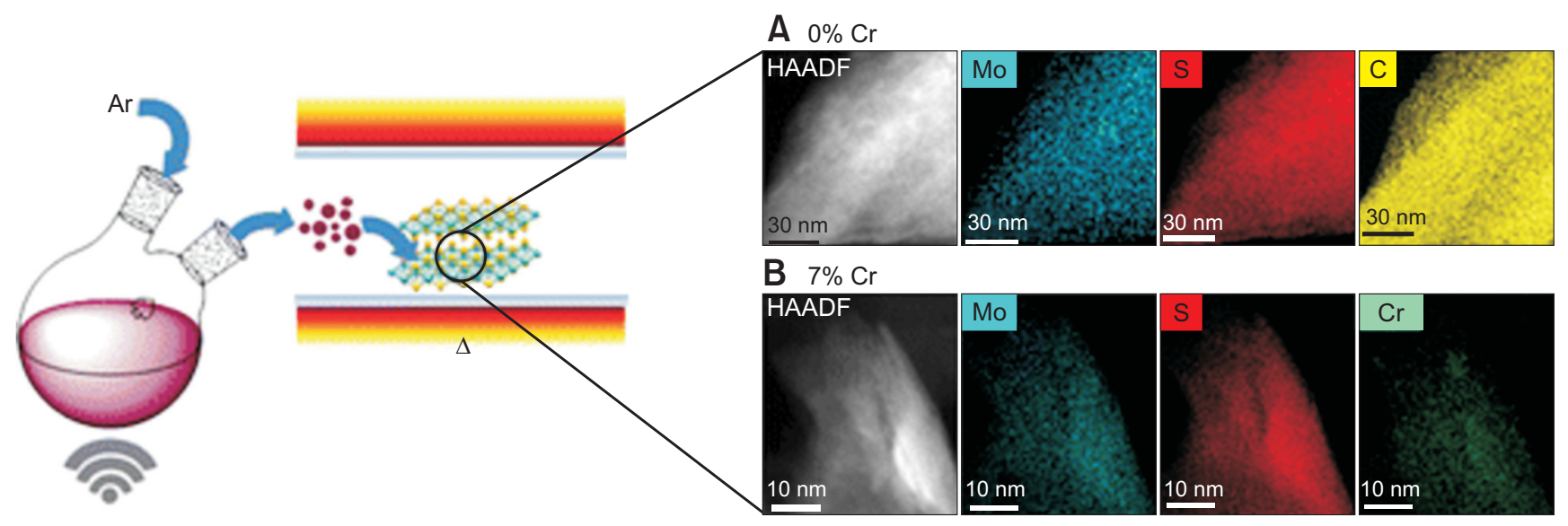

Fig. 6. Schematic of aerosol-assisted chemical vapor deposition (AACVD) and high-angle annular dark field scanning transmission electron microscopy imaging with energy dispersive X-ray spectroscopy spectra of (A) undoped $\left(0 \mathrm{~mol} \mathrm{\%} \mathrm{Cr}, 450^{\circ} \mathrm{C}\right)$ and (B) $\mathrm{Cr}$-doped $\left(7.1 \mathrm{~mol} \% \mathrm{Cr}, 450^{\circ} \mathrm{C}\right) \mathrm{MoS}_{2}$ nanosheets synthesized via AACVD. Reprinted from Lewis et al. (2015) (Chem. Mater. 27, 1367-1374) with permission of American Chemical Society.

(EDS) mapping (Tedstone et al., 2015).

Thin film rhenium disulfide $\left(\mathrm{ReS}_{2}\right)$ and $\mathrm{Mo}_{1-\mathrm{x}} \mathrm{Re}_{\mathrm{x}} \mathrm{S}_{2}$ alloys were also successfully synthesized via AACVD according to a study by Al-Dulaimi et al. (2016). The precursor was synthesized with $\left[\operatorname{Re}(\mu-\mathrm{SiPr})_{3}(\mathrm{SiPr})_{6}\right]$ and $\left[\mathrm{Mo}\left(\mathrm{S}_{2} \mathrm{CNEt}_{2}\right)_{4}\right]$ at certain molar ratios in $40 \mathrm{~mL}$ of THF at $475^{\circ} \mathrm{C}$. The created precursor mist was transported by Ar gas to the furnace, and the decomposed precursors were deposited on the glass substrates.

\section{Chemical Doping}

Chemical doping is a method in which 2D materials are doped effectively by simply soaking them in dopant solution or by dropping dopant solution. It is possible to tune the properties of the materials by chemical doping because of the charge transfer between the dopant solution and the materials.

Yang et al. (2014) reported chemical doping by soaking mechanically exfoliated TMDs in a solution. They fabricated chlorine $(\mathrm{Cl})$-doped $\mathrm{WS}_{2}$ and $\mathrm{MoS}_{2}$ back-gate FET as shown in Fig. 7A, by exfoliating a few-layers of TMDs with scotch tape and soaking them in undiluted 1,2-dichloroethane (DCE) for over $12 \mathrm{~h}$ at room temperature. The contact resistance of a metal-semiconductor junction was significantly reduced from $500 \mathrm{k} \Omega \cdot \mu \mathrm{m}$ for undoped $\mathrm{WS}_{2}$ to $0.7 \mathrm{k} \Omega \cdot \mu \mathrm{m}$ for $\mathrm{Cl}$-doped $\mathrm{WS}_{2}$, as shown in Fig. 7B. This was found to improve various properties of the FET, such as its drain current, on/off ratio, and mobility by $\mathrm{Cl}$ doping on TMDs.

Moreover, molecule-doped TMDs are being actively studied. Mouri et al. (2013) suggested the possibility of molecular 
A

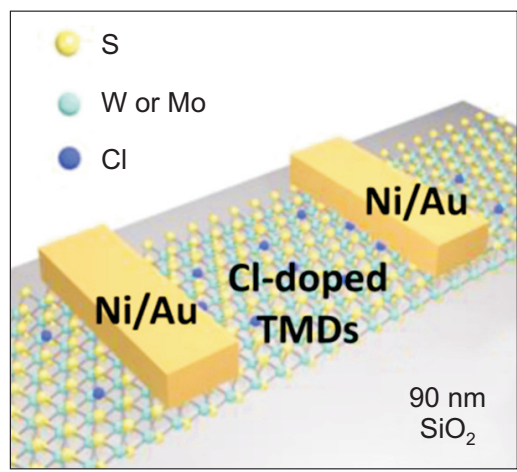

B

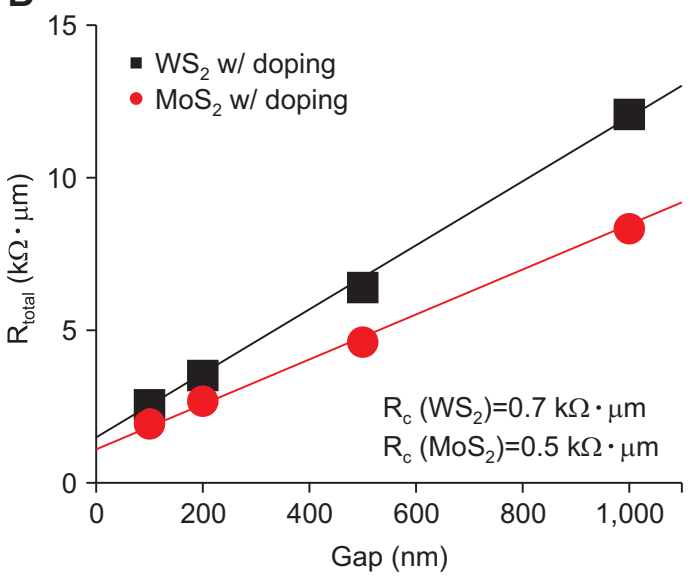

Fig. 7. (A) Schematic of a back-gate field effect transistor in which chlorine $(\mathrm{Cl})$ doped transition metal dichalcogenides (TMDs) are used as channel materials. (B) Contact resistances $\left(\mathrm{R}_{\mathrm{c}}\right)$ of $0.7 \mathrm{k} \Omega \cdot \mu \mathrm{m}$ for $\mathrm{Cl}$ doped- $\mathrm{WS}_{2}$ and $0.5 \mathrm{k} \Omega \cdot \mu \mathrm{m}$ for $\mathrm{Cl}$ doped $\mathrm{MoS}_{2}$ under $50 \mathrm{~V}$ of back-gate bias. Reprinted from Yang et al. (2014) (Nano Lett. 14, 6275-6280) with permission of American Chemical Society.
A

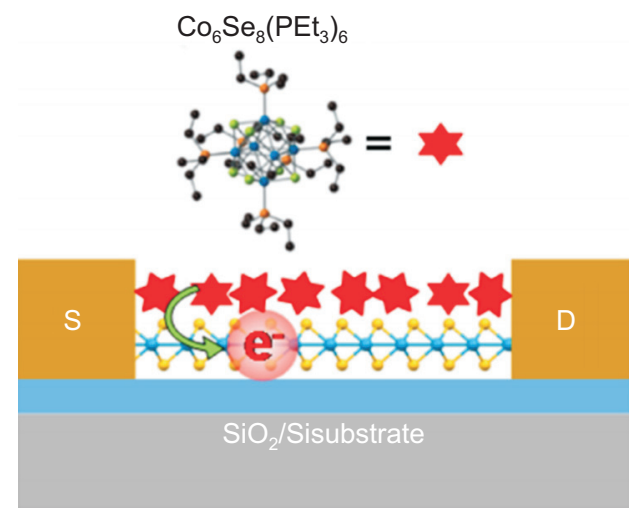

C

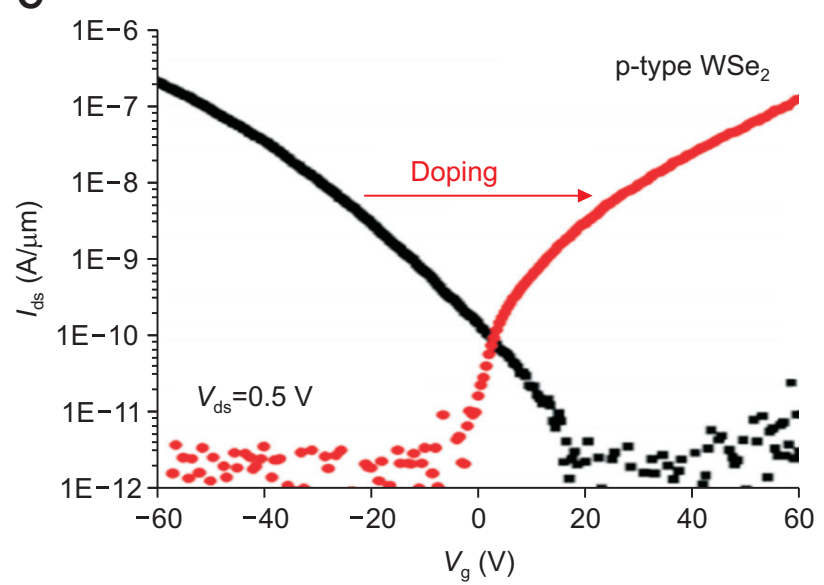

B

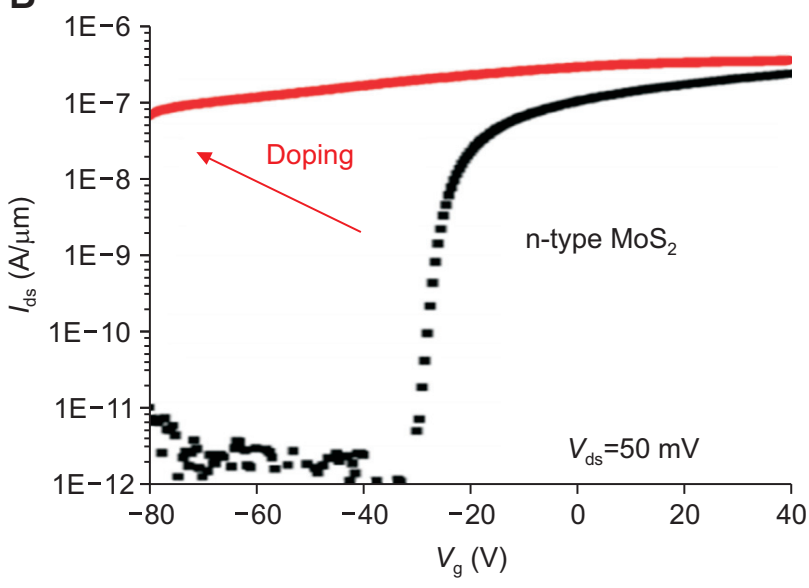

D

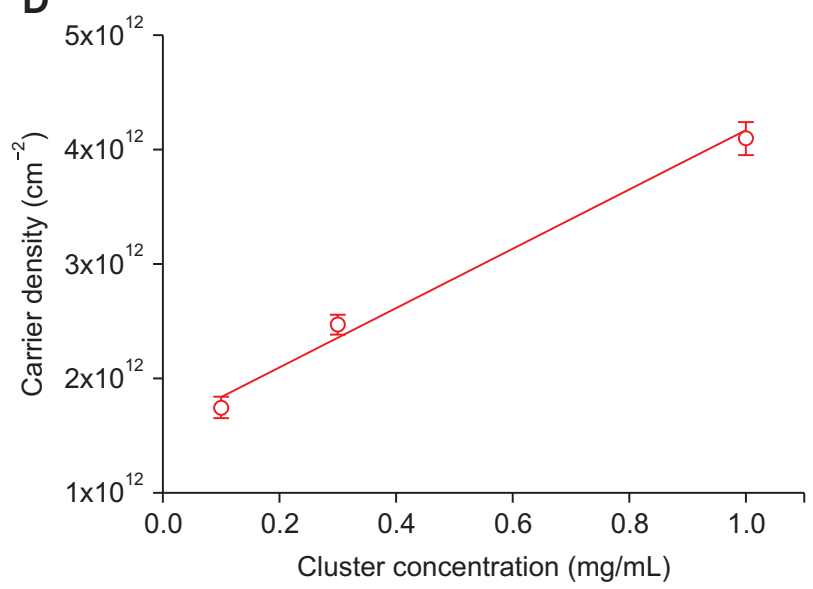

Fig. 8. (A) Schematic of a fabricated back-gate field effect transistor with superatom (electron-rich molecular cluster)-doped few-layer $\mathrm{MoS}_{2}$ and $\mathrm{WSe}_{2}$ applied for channels. The differences in the transfer characteristics are illustrated for pristine and superatom-doped (B) few-layer $\mathrm{MoS}_{2}$ and $(\mathrm{C})$ few-layer $\mathrm{WSe}_{2}$. (D) The carrier density of superatom-doped bilayer WSe $\mathrm{W}_{2}$ depends on the cluster concentration in solution. Reprinted from Yu et al. (2016) (Nano Lett. 16, 3385-3389) with permission of American Chemical Society.

doping on monolayer $\mathrm{MoS}_{2}$ via chemical doping, and they verified the enhanced performance of the material using PL spectra. The molecular dopant solutions were prepared as
$0.02 \mu \mathrm{mol} / \mathrm{mL}$ of 2,3,5,6-tetrafluoro-7,7,8,8-tetracyanoquinodimethane $\left(\mathrm{F}_{4} \mathrm{TCNQ}\right)$ and 7,7,8,8-tetracyanoquinodimetha ne (TCNQ) for p-type chemical dopant solutions, whereas 
nicotinamide adenine dinucleotide (NADH) was used in an n-type dopant solution. Then, $10 \mu \mathrm{L}$ of dopant solution was dropped on the prepared exfoliated $\mathrm{MoS}_{2}$. The same doping sequence was repeated after the solutions evaporated completely. The PL intensity was drastically enhanced for p-type doped $\mathrm{MoS}_{2}$ with $\mathrm{F}_{4} \mathrm{TCNQ}$ and TCNQ. However, it decreased for n-type doped $\mathrm{MoS}_{2}$ with NADH.

$\mathrm{Yu}$ et al. (2016) reported the synthesis of superatom-doped $\mathrm{MoS}_{2}$ and $\mathrm{WSe}_{2}$ via a chemical doping method. The superatom, $\mathrm{Co}_{6} \mathrm{Se}_{8}\left(\mathrm{PEt}_{3}\right)_{6}$, is an electron-rich octahedral dopant as shown in Fig. 8A. The fabricated FETs assembled with exfoliated TMDs and metal electrodes were immersed in dopant solutions, in which the superatoms were dissolved in toluene, for $10 \mathrm{~min}$. And then, the FETs were rinsed with fresh toluene. As shown in Fig. 8B-D, the performance of FET channels containing superatom-doped $\mathrm{MoS}_{2}$ and $\mathrm{WS}_{2}$ was significantly enhanced because the superatoms effectively transferred electrons to the TMD films.

Furthermore, molecular doping with particular functional groups on the TMDs was studied by Sim et al. (2015). Mechanically exfoliated $\mathrm{MoS}_{2}$ was annealed in a tube furnace at $250^{\circ} \mathrm{C}$ for $1 \mathrm{~h}$ to create vacancies at the $\mathrm{S}$ sites of $\mathrm{MoS}_{2}$, as shown in Fig. 9A. Then, the vacancy-induced $\mathrm{MoS}_{2}$ was soaked in $1 / 40(\mathrm{v} / \mathrm{v})$ mercaptoethylamine $(\mathrm{NH} 2$-terminated thiol, MEA)/ethanol and $1 \mathrm{H}, 1 \mathrm{H}, 2 \mathrm{H}, 2 \mathrm{H}$-perfluorodecanethiol $\left(\mathrm{CF}_{3}\right.$-terminated thiol, FDT)/THF solution for $72 \mathrm{~h}$ to dope the molecular dopants at the vacancy sites, as illustrated in Fig. 9B; the molecular structures of the dopants are shown in Fig. 9C. The electrical properties of $\mathrm{MoS}_{2}$ were tuned on the basis of the molecular dopants, which had particular functional groups, as shown in Fig. 9D and E.

\section{Other Synthesis Methods}

Additional methods of doping have been investigated. Onepot synthesis of target materials are widely applied; this is because the reactions occur in one vessel and purification of the chemical compounds is readily achieved (Hayashi, 2016). In addition, this method saves time and resources while providing increased chemical yield. Deng et al. (2015) obtained platinum (Pt)-doped $\mathrm{MoS}_{2}$ via a one-pot chemical synthesis. First, the precursors, $900 \mathrm{mg}$ of $\left(\mathrm{NH}_{4}\right)_{6} \mathrm{Mo}_{7} \mathrm{O}_{24} \cdot 4 \mathrm{H}_{2} \mathrm{O}$ and $0.442 \mathrm{~mL}\left(0.19 \mathrm{~mol} \cdot \mathrm{L}^{-1}\right)$ of $\mathrm{H}_{2} \mathrm{PtCl}_{6}(\mathrm{aq})$, were mixed and dissolved in $20 \mathrm{~mL}$ of deionized water. Then, the solution and $10 \mathrm{~mL}$ of $\mathrm{CS}_{2}$ were transferred to a stainless-steel autoclave
A
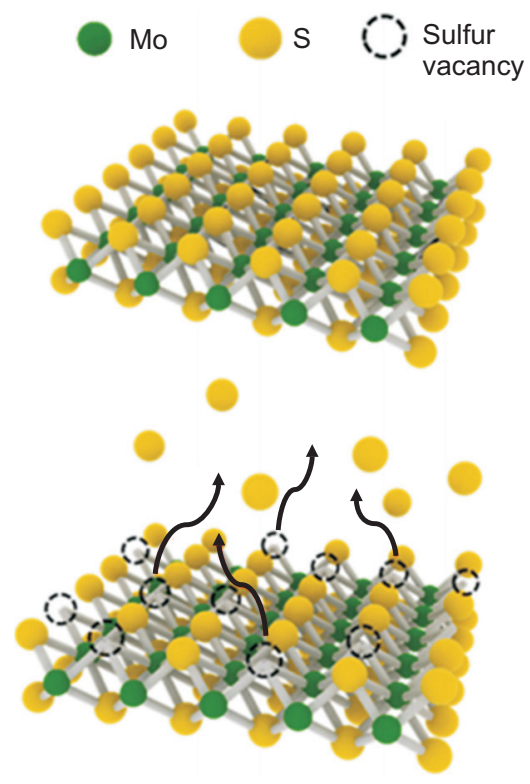

B

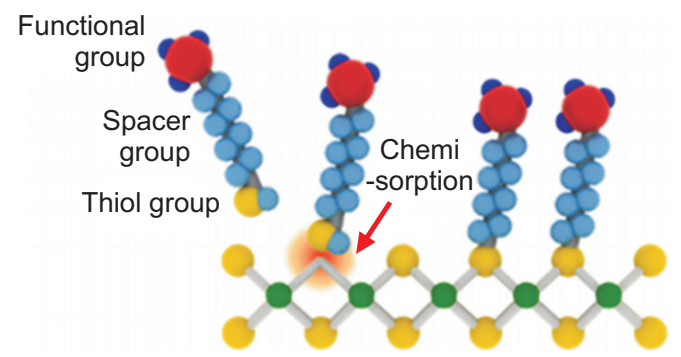

C

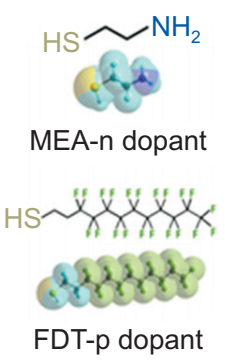

E

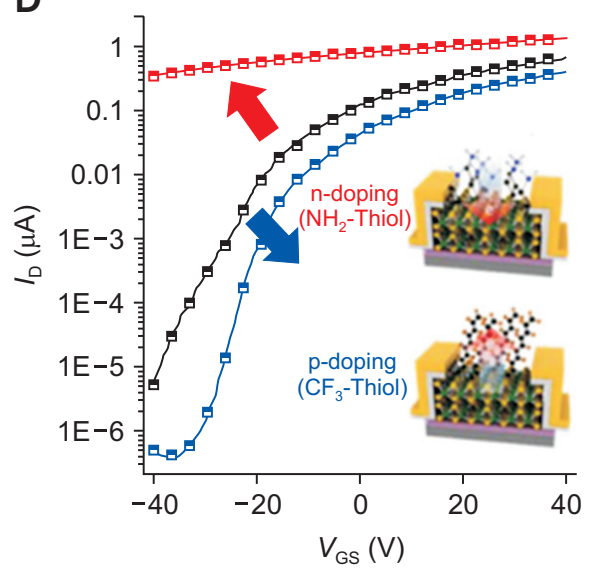

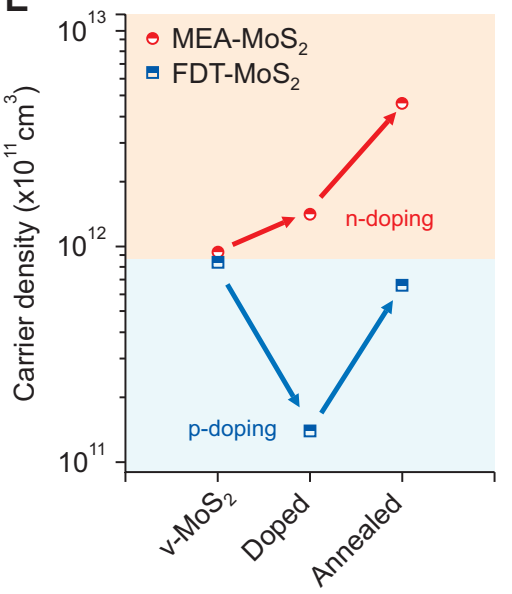

Fig. 9. Schematic of (A) vacancy-induced $\mathrm{MoS}_{2}$ under annealing at $250^{\circ} \mathrm{C}$ for $1 \mathrm{~h}$ and (B) chemisorption of thiol-functionalized molecules at the $\mathrm{S}$ vacancy sites of $\mathrm{MoS}_{2}$. (C) Structures of two molecular dopants which terminated with thiol group containing $\mathrm{NH}_{2}$ (MEA; n-dopant) and fluorocarbon group (FDT; p-dopant). (D) The transfer characteristics of fabricated back-gated $\mathrm{MoS}_{2}$ FETs with undoped (black line), MEA-doped (red line), and FDT-doped (blue line) $\mathrm{MoS}_{2}$. (E) Carrier density difference of $\mathrm{MoS}_{2}$ before and after the doping process with no gate voltage. Reprinted from Sim et al. (2015) (ACS Nano 9, 12115-12123) with permission of American Chemical Society. 
and annealed at $400^{\circ} \mathrm{C}$ for $4 \mathrm{~h}$. The product was then stirred with saturated $\mathrm{NaOH}(\mathrm{aq})$ at $60^{\circ} \mathrm{C}$ for $3 \mathrm{~h}$. Finally, the product was washed several times with deionized water and ethanol and dried thoroughly at $100^{\circ} \mathrm{C}$. The extended X-ray absorption fine structure and HAADF-STEM analysis showed that $\mathrm{Pt}$ was doped as a single atom at the substitutional Mo sites, as shown in Fig. 10A-D. The Pt-doped few-layer $\mathrm{MoS}_{2}$ that was synthesized by this one-pot method showed significantly enhanced hydrogen evolution reaction (HER) catalytic activity compared to pristine $\mathrm{MoS}_{2}$. This is because Pt doping increased the catalytic activity of in-plane $\mathrm{MoS}_{2}$, while pristine $2 \mathrm{D} \mathrm{MoS}_{2}$ only shows catalytic activity at its edge sites. The Pt-doped $\mathrm{MoS}_{2}$ exhibited better HER activity than blank glassy carbon (GC), bulk $\mathrm{MoS}_{2}$, and few-layer $\mathrm{MoS}_{2}$. However, its performance was inferior to that of $40 \% \mathrm{Pt} / \mathrm{C}$ catalyst, as shown in Fig. 10E. Furthermore, $\mathrm{MoS}_{2}$ doped with other transition metals is also expected to show enhanced
HER activity according to DFT calculations, as shown in Fig. 10F.

Qin et al. (2014) reported the synthesis of nitrogen (N)-doped $\mathrm{MoS}_{2}$ nanosheets by the sol-gel method. The sol-gel method is a relatively simple and cost-efficient method to produce solid materials from small molecules on a large scale. The dispersed monomers in the sol are transformed to a gel phase by coating, gelling, or precipitation. Mixed $0.5 \mathrm{~g}$ molybdenum chloride $\left(\mathrm{MoCl}_{5}\right)$ and thiourea $\left(\left(\mathrm{NH}_{2}\right)_{2} \mathrm{CS}\right)$ in a particular molar ratio were dissolved in ethanol under stirring, and the dried solution was transformed to a brown gel powder. This powder was heated in a quartz tube for $3 \mathrm{~h}$ at different temperatures under Ar gas flow. This research is remarkable because it provides a new method to dope non-metal dopants on TMDs, and the performance of the N-doped $\mathrm{MoS}_{2}$ was excellent.
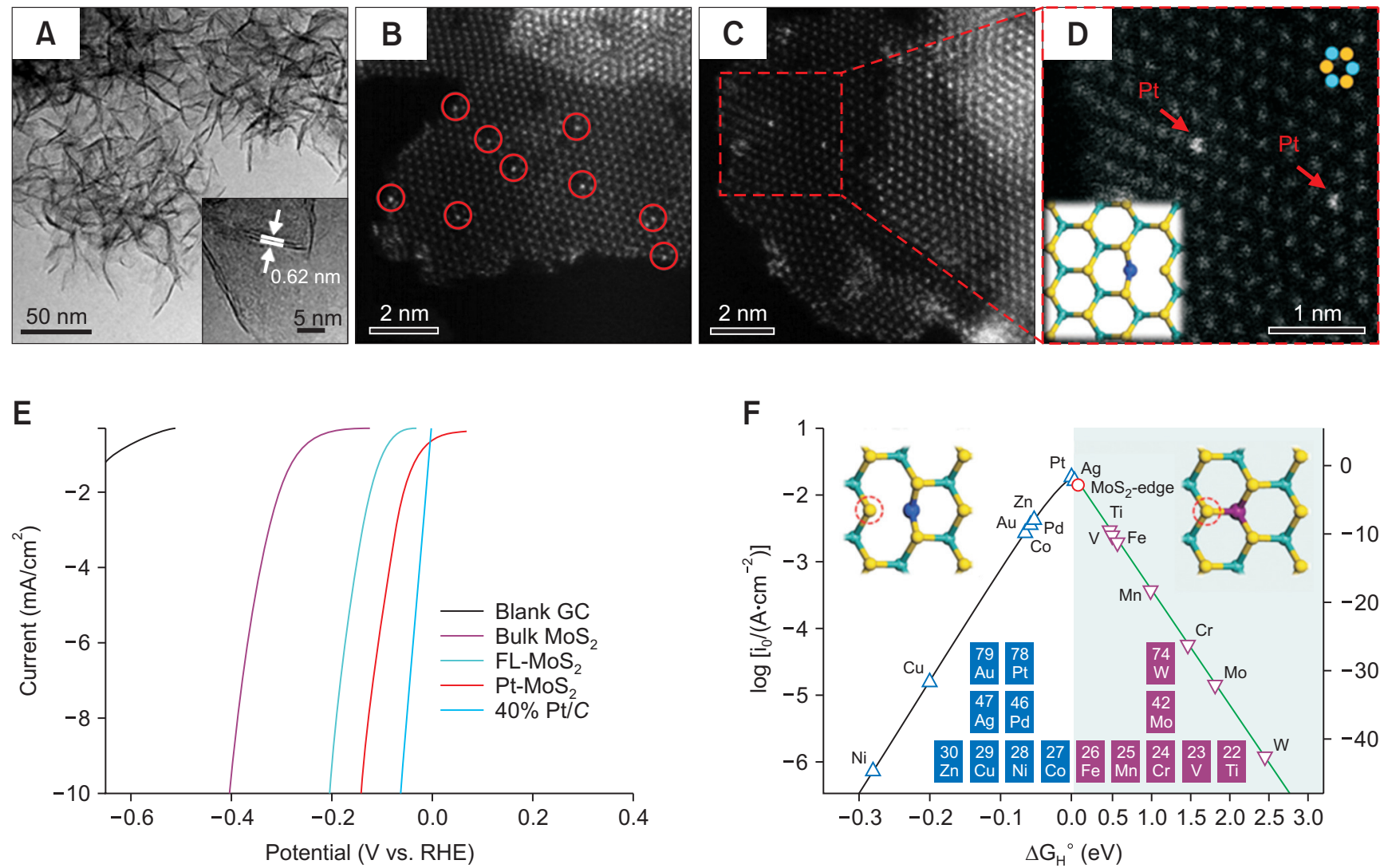

Fig. 10. (A) Transmission electron microscopy (TEM) image and inset showing a layer distance of Pt-doped $\mathrm{MoS}_{2}$ and (B,C) high-angle annular dark field scanning TEM images of uniformly doped Pt on $\mathrm{MoS}_{2}$ with a honeycomb arrangement. The brighter contrast points are Pt doped in the substitutional sites of Mo. The magnified red dashed square in (C) is illustrated in (D). The inset of (D) is simulated configuration of Pt-doped MoS $\mathrm{S}_{2}$ and each green, yellow and blue balls represent Mo, S and Pt, respectively. (E) Hydrogen evolution reaction (HER) polarization curve of Pt-doped MoS ${ }_{2}$, as the red line, compared with blank glassy carbon (GC) electrode, bulk $\mathrm{MoS}_{2}$, few-layer (FL) MoS 2 without doping, and the traditional catalyst $40 \% \mathrm{Pt} / \mathrm{C}$. The potentials are calibrated with a reversible hydrogen electrode (RHE). (F) Density functional theory calculations of transition metal-doped $\mathrm{MoS}_{2}$ with a particular configuration that is coordinated with $4 \mathrm{~S}$ bonds (left) and $6 \mathrm{~S}$ bonds (right). The relationship between current $\left(\log \left(\mathrm{i}_{0}\right)\right)$ and adsorption free energy $\left(\Delta \mathrm{G}_{\mathrm{H}}{ }^{\circ}\right)$, which shows the possibility of other dopants for HER catalysts. Reproduced from Deng et al. (2015) (Energy Environ. Sci. 8, 1594-1601) with permission of The Royal Society of Chemistry. 


\section{CONCLUSIONS}

Various synthesis methods for doping on 2D TMDs have been investigated, including CVT, in situ doping via CVD, AACVD, and chemical doping. Also, in addition to transition metals such as $\mathrm{Nb}, \mathrm{Re}, \mathrm{Mn}$, and $\mathrm{Au}$, non-transition metals such as $\mathrm{N}$ and $\mathrm{Cl}$ have been doped on 2D TMDs through diverse doping methods. Furthermore, molecular doping on 2D TMDs has been extensively studied using molecular dopant solutions such as MEA, FDT, and a superatom. Molecular doping via chemical doping is convenient, efficient and greatly enhances the performance of 2D TMDs. Through doping on 2D TMDs, properties such as band structure, catalytic activity, morphology, and electronic and optic properties are significantly improved; however, it is highly influenced by the species and concentration of the dopants and by the synthesis method. Therefore, selecting the proper synthesis methods and dopants are important for improving the performance of 2D TMDs. To characterize and evaluate the performance of doped TMDs, the doping sites are generally observed using TEM images and X-ray photoelectron spectroscopy; the uniformity of the doping is identified by EDS. The improvements in the performance of doped TMDs is analyzed through PL spectra and fabricated FET devices by examining their contact resistance, transfer characteristics, carrier density, etc. By doping 2D TMDs, their applications as optical devices, electrical devices, and catalysts can be expanded significantly.

In addition to doping, other chemical and physical tuning methods of 2D TMDs are being actively studied. Studies of the strong excitonic effects and spin- and valley-dependent properties of 2D TMDs have recently begun. Additionally, further research may extend the field of 2D TMDs from 2D binary TMDs into ternary phase TMDs, such as molybdenum tungsten disulfide $\left(\mathrm{MoWS}_{2}\right)$ and Molybdenum sulfide selenide (MoSSe) and so on. These ternary phase TMD alloys have recently received attention because they have superior intermiscibility and good thermodynamic stability. Also, these ternary phase TMDs are expected to greatly expand the possible applications of TMDs because the band gaps of $2 \mathrm{D}$ ternary phase TMDs can be simply engineered by controlling the compositions of the materials. Therefore, it is predicted that they will be applied in next-generation optoelectronic, ferromagnetic, semiconducting, and spintronic devices.

\section{CONFLICT OF INTEREST}

No potential conflict of interest relevant to this article was reported.

\section{ACKNOWLEDGMENTS}

This work was supported by the National Research Foundation of Korea (NRF) grant funded by the Korea government (MSIP) (2015R1A2A2A01006992) and Nano Material Technology Development Program (2012M3A7B4049807).

\section{REFERENCES}

Al-Dulaimi N, Lewis D J, Zhong X L, Malik M A, and O'Brien P (2016) Chemical vapour deposition of rhenium disulfide and rhenium-doped molybdenum disulfide thin films using single-source precursors. $J$. Mater. Chem. C 4, 2312-2318.

Ataca C, Sahin H, and Ciraci S (2012) Stable, single-layer MX2 transitionmetal oxides and dichalcogenides in a honeycomb-like structure. J. Phys. Chem. C 116, 8983-8999.

Binnewies M, Glaum R, Schmidt M, and Schmidt P (2013) Chemical vapor transport reactions-a historical review. ZAAC 639, 219-229.

Deng J, Li H, Xiao J, Tu Y, Deng D, Yang H, Tian H, Li J, Ren P, and Bao $X$ (2015) Triggering the electrocatalytic hydrogen evolution activity of the inert two-dimensional MoS2 surface via single-atom metal doping. Energy Environ. Sci. 8, 1594-1601.

Gao J, Kim Y D, Liang L, Idrobo J C, Chow P, Tan J, Li B, Li L, Sumpter B G, and Lu T M (2016) Transition-metal substitution doping in synthetic atomically thin semiconductors. Adv. Mater. 28, 9735-9743.

Hayashi Y (2016) Pot economy and one-pot synthesis. Chem. Sci. 7, 866880.

Hwang N M (2016). Non-Classical Crystallization of Thin Films and Nanostructures in CVD and PVD Processes (Vol. 60) (Springer,
Seoul).

Lewis D J, Tedstone A A, Zhong X L, Lewis E A, Rooney A, Savjani N, Brent J R, Haigh S J, Burke M G, and Muryn C A (2015) Thin films of molybdenum disulfide doped with chromium by aerosol-assisted chemical vapor deposition (AACVD). Chem. Mater. 27, 1367-1374.

Lin Y C, Dumcenco D O, Komsa H P, Niimi Y, Krasheninnikov A V, Huang Y S, and Suenaga K (2014) Properties of individual dopant atoms in single-layer MoS2: atomic structure, migration, and enhanced reactivity. Adv. Mater. 26, 2857-2861.

Mak K F, He K, Lee C, Lee G H, Hone J, Heinz T F, and Shan J (2013) Tightly bound trions in monolayer MoS2. Nat. Mater. 12, 207-211.

Mouri S, Miyauchi Y, and Matsuda K (2013) Tunable photoluminescence of monolayer MoS2 via chemical doping. Nano Lett. 13, 5944-5948.

Novoselov K S, Geim A K, Morozov S V, Jiang D, Zhang Y, Dubonos S V, Grigorieva I V, and Firsov A A (2004) Electric field effect in atomically thin carbon films. Science 306, 666-669.

Park J H and Sudarshan T S (2001) Chemical Vapor Deposition (Vol. 2) (ASM International, Illinois).

Qin S, Lei W, Liu D, and Chen Y (2014) In-situ and tunable nitrogendoping of MoS2 nanosheets. Sci. Rep. 4, 7582. 
Sim D M, Kim M, Yim S, Choi M J, Choi J, Yoo S, and Jung Y S (2015) Controlled doping of vacancy-containing few-layer MoS2 via highly stable thiol-based molecular chemisorption. ACS Nano 9, 1211512123.

Suh J, Park T E, Lin D Y, Fu D, Park J, Jung H J, Chen Y, Ko C, Jang C, and Sun $Y$ (2014) Doping against the native propensity of MoS2: degenerate hole doping by cation substitution. Nano Lett. 14, 69766982.

Tedstone A A, Lewis D J, Hao R, Mao S M, Bellon P, Averback R S, Warrens C P, West K R, Howard P, and Gaemers S (2015) Mechanical properties of molybdenum disulfide and the effect of doping: an in situ TEM study. ACS Appl. Mater. Interfaces 7, 20829-20834.

Tedstone A A, Lewis D J, and O'Brien P (2016) Synthesis, properties, and applications of transition metal-doped layered transition metal dichalcogenides. Chem. Mater. 28, 1965-1974.

Wang H, Yuan H, Hong S S, Li Y, and Cui Y (2015) Physical and chemical tuning of two-dimensional transition metal dichalcogenides. Chem Soc. Rev. 44, 2664-2680.

Wang Q H, Kalantar-Zadeh K, Kis A, Coleman J N, and Strano M S (2012) Electronics and optoelectronics of two-dimensional transition metal dichalcogenides. Nat. Nanotechnol. 7, 699-712.

Yang L, Majumdar K, Liu H, Du Y, Wu H, Hatzistergos M, Hung P, Tieckelmann R, Tsai W, and Hobbs C (2014) Chloride molecular doping technique on 2D materials: WS2 and MoS2. Nano Lett. 14, 6275-6280.

Yu J, Lee C H, Bouilly D, Han M, Kim P, Steigerwald M L, Roy X, and Nuckolls $C$ (2016) Patterning superatom dopants on transition metal dichalcogenides. Nano Lett. 16, 3385-3389.

Zhang K, Feng S, Wang J, Azcatl A, Lu N, Addou R, Wang N, Zhou C, Lerach J, and Bojan V (2015) Manganese doping of monolayer MoS2: the substrate is critical. Nano Lett. 15, 6586-6591. 